\title{
Riesgo cardiovascular asociado a menopausia
}

\section{Cardiovascular risk associated to menopause}

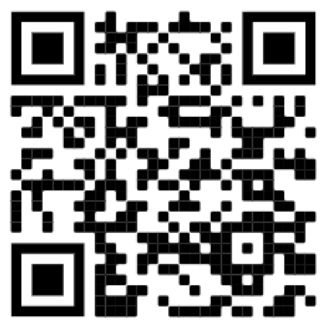

\author{
${ }^{1}$ Dr. Bryan Daniel Bolaños Chaves \\ Investigador independiente, Alajuela, Costa Rica \\ (C) https://orcid.org/0000-0003-2833-980X
}

${ }^{2}$ Dra. Estefanía Ortega Muñoz Investigadora independiente, San José, Costa Rica b.tps://orcid.org/0000-0002-7810-4649

\begin{abstract}
RECIBIDO
$26 / 10 / 2020$

${ }^{1}$ Médico general, graduado de la Universidad de Iberoamerica (UNIBE), cod. MED16428. bdbolanos18@gmail.com

$\mathbf{2}$ Médico general, graduado de la Universidad de lberoamerica (UNIBE), cod. MED16447. dra.ortegam@gmail.com

CORREGIDO ACEPTADO

$15 / 11 / 2020$

$30 / 11 / 2020$

\section{RESUMEN}

La enfermedad cardiovascular representa una de las principales causas de mortalidad en mujeres postmenopáusicas. Posterior a la menopausia, se presentan múltiples factores que aumentan el riesgo real de enfermedad cardiovascular, dentro de los cuales se incluye el tabaquismo, la hipertensión arterial, las dislipidemias, la diabetes mellitus tipo dos, la edad de la menarca y de la menopausia, así como los síntomas vasomotores, además de factores de riesgo tradicionales del periodo de transición, tales como la disminución de la tolerancia a la glucosa y la disfunción endotelial.Asimismo, se incluyen otros factores, como la disfunción ovárica primaria y la menopausia inducida quirúrgicamente, que suelen adelantar todos los procesos fisiológicos de una menopausia natural, resultando en un importante aumento del riesgo cardiovascular.

PALABRAS CLAVE: menopausia; enfermedades cardiovasculares; enfermedad coronaria; menopausia prematura; terapia de reemplazo de estrógeno.

\section{ABSTRACT}

Cardiovascular disease represents one of the main causes of mortality in postmenopausal women. After menopause, there are many factors that increase the real risk of cardiovascular disease, including smoking, high blood pressure, dyslipidemias, type two diabetes mellitus, age at menarche and menopause, and vasomotor symptoms, in addition, the traditional risk factors of the transition period, such as decreased glucose tolerance and endothelial dysfunction. Also, other factors are included, such as primary ovarian dysfunction and surgical menopause, which usually advance all the
\end{abstract}


physiological processes of a natural menopause, resulting in a significant increase in cardiovascular risk.

KEYWORDS: menopause; cardiovascular diseases; coronary disease; premature menopause; estrogen replacement therapy.

\section{INTRODUCCIÓN}

La menopausia natural se define como la ausencia de menstruación durante un período de doce meses, la cual ocurre generalmente entre los 49 y 52 años. Un $5 \%$ de las mujeres desarrolla una menopausia precoz (entre los 40 y 45 años de edad) y aproximadamente un $1 \%$ presenta una menopausia prematura (antes de los 40 años de edad), también conocida como insuficiencia ovárica primaria (1). La aparición de la menopausia supone en la mujer un aumento significativo del riesgo de presentar eventos cardiovasculares; una gran variedad de síntomas tales como los vasomotores (sofocos y sudoración nocturna), depresión, ansiedad, crisis de pánico, irritabilidad, fatiga, disminución de la libido e insomnio, son comunes en mujeres postmenopáusicas y se han asociado a un aumento del riesgo cardiovascular (2). Asimismo, se ha estudiado a fondo el rol que juegan otros factores como la edad, las hormonas tanto endógenas como exógenas, y otros factores ambientales como el tabaco, en el verdadero aumento de la patología cardiovascular en mujeres postmenopáusicas. Conforme la medicina avanza, cada día se revela más información en todos los frentes de investigación y sin duda, los factores de riesgo cardiovascular; siendo sus patologías, la primera causa de muerte no traumática en el mundo, no se queda atrás. Actualmente se publican estudios de forma diaria con datos cuantificables, exponiendo nuevos factores de riesgo cardiovascular asociados directamente con la menopausia, lo cual contribuye de forma significativa a la comprensión de este proceso y, por tanto, a desarrollar nuevas estrategias para controlar $\mathrm{y}$ disminuir el riesgo.

El objetivo del presente estudio es establecer el riesgo cardiovascular real asociado a la menopausia, evaluando la mayor cantidad de investigaciones recientes con alta confiabilidad que identifiquen los factores de riesgo individuales, ya sean hormonales, alimenticios, quirúrgicos, genéticos, ambientales, entre otros, que, en conjunto, sumen el ya conocido aumento de riesgo cardiovascular en mujeres postmenopáusicas.

\section{MÉTODOS}

Para la elaboración de esta investigación, se realizaron búsquedas en las bases de datos Cochrane, Pubmed y Google Académico, con las siguientes palabras clave; "menopausia" y "factores de riesgo cardiaco", tras lo cual se establecieron y aplicaron los siguientes criterios de inclusión; "publicados de 2015 en adelante", "artículos en español", "artículos en inglés", y los siguientes criterios de exclusión "artículos en idiomas diferentes a inglés o español", "artículos publicados antes del 2015", "artículos respecto a investigación inconclusas 0 sin resultados aún publicados"; tras lo cual se obtuvieron quince fuentes bibliográficas satisfactorias. 


\section{EPIDEMIOLOGÍA}

La enfermedad cardiovascular representa una de las principales causas de mortalidad en mujeres postmenopáusicas, principalmente la enfermedad coronaria ateroesclerótica. Previo a la menopausia, el riesgo cardiovascular es inferior al de los hombres; y por lo general, las mujeres postmenopáusicas desarrollan la enfermedad 10 años después que los hombres; esto está relacionado con la disminución de las concentraciones de hormonas ováricas durante la transición menopáusica, que se asocia a una mayor prevalencia de múltiples factores de riesgo cardiovascular, tales como diabetes mellitus tipo dos, dislipidemias, síndrome metabólico, cambios desfavorables en el peso y distribución de grasa corporal, sensibilidad insulínica y del tono simpático, entre otros $(3,4)$.

Puesto que las mujeres tienen una mayor esperanza de vida que los hombres, el número absoluto de muertes por enfermedades cardiovasculares es mayor en mujeres que en hombres y, además, se ha observado tasas de mortalidad en mujeres de menos de 55 años de edad (5).

\section{FISIOPATOLOGÍA}

Diversos mecanismos biológicos pueden explicar la asociación entre hormonas sexuales endógenas y el riesgo cardiovascular. Los estrógenos promueven la vasodilatación mediante el aumento del óxido nítrico plasmático, e inhiben el sistema renina angiotensina mediante la disminución de la transcripción de la enzima convertidora de angiotensina. Además de su favorable efecto sobre los lípidos, los estrógenos también pueden disminuir la presión arterial mediante el aumento de la vasodilatación endotelial y modulando la función autonómica. Además, los estrógenos regulan los marcadores inflamatorios específicos y citoquinas.

En un estudio observacional en el cual participaron mujeres postmenopáusicas sin enfermedad cardiovascular de base, se asoció una relación testosterona / estradiol alta con un riesgo elevado de enfermedades cardiovasculares. Asimismo, los niveles de testosterona total altos se relacionaron con un mayor riesgo de enfermedad coronaria y los niveles de estradiol altos con un menor riesgo de enfermedad coronaria. Las asociaciones persistieron después del ajuste por factores de riesgo cardiovascular tradicionales, lo que sugiere un papel independiente de las hormonas sexuales en los eventos cardiovasculares (6).

\section{FACTORES DE RIESGO CARDIOVASCULAR}

Dentro de los factores de riesgo cardiovasculares en mujeres postmenopáusicas se incluyen los siguientes:

- Tabaquismo: el fumado continúa siendo uno de los factores de riesgo más importantes pero prevenibles de las enfermedades cardiovasculares ateroescleróticas. A nivel mundial, más hombres que mujeres son fumadores en la mayoría de los países, sin embargo, existe más equivalencia entre géneros en Europa y América (3).

- Hipertensión arterial: es un factor de riesgo importante tanto en mujeres como en hombres. En los países desarrollados, el $30 \%$ de las mujeres padecen de hipertensión arterial y esta prevalencia es incluso mayor en los 
países de ingresos medios-bajos, alcanzando hasta el $53 \%$.

La prevalencia de hipertensión arterial en mujeres postmenopáusicas es más del doble de la de las mujeres premenopáusicas, por tanto, la monitorización de la presión arterial es imprescindible en mujeres de todas las edades y un control eficaz de la presión arterial en el período premenopáusico, en la transición menopáusica o en la postmenopausia temprana evitará el desarrollo de enfermedad cardiovascular en una edad avanzada (3).

- Dislipidemias: la hipercolesterolemia representa la principal causa de enfermedad cardiovascular en ambos sexos y su tratamiento se ha asociado a reducciones significativas de la morbilidad y la mortalidad (3).

Después de la menopausia, el colesterol total y las lipoproteínas de baja densidad (LDL) suelen aumentar, y estos cambios vienen acompañados de una disminución de las lipoproteínas de alta densidad (HDL), la cual es un predictor de mortalidad cardiovascular en mujeres. Al mismo tiempo, los triglicéridos elevados son un factor de riesgo independiente de mortalidad por cardiopatía coronaria en mujeres, especialmente en mujeres con concentraciones bajas de colesterol HDL. Esto explica parcialmente el aumento del riesgo cardiovascular en mujeres postmenopáusicas, particularmente aquellas con un inicio más temprano de la menopausia $(3,7)$. En un estudio transversal donde se compararon los factores de riesgo cardiovascular entre mujeres de la misma edad, con diferentes estados menopáusicos, se demostró que, en todas las edades entre 46 y 55 años, las mujeres postmenopáusicas naturales presentaron un colesterol total y un colesterol LDL más alto en comparación con las mujeres premenopáusicas del mismo rango de edad (8).

- Diabetes mellitus tipo 2: está relacionada con una mayor morbilidad y mortalidad por enfermedad cardiovascular tanto en hombres como en mujeres; sin embargo, las mujeres con diabetes parecen tener un riesgo relativo mayor de enfermedad cardiovascular que los hombres (6). Asimismo, las tasas de prevalencia crecientes de diabetes mellitus tipo 2 se han atribuido al envejecimiento, estilos de vida asociados a la obesidad, que predispone a diversas alteraciones metabólicas ligadas a la resistencia a la insulina (7).

La mayoría de los estudios demuestran un mayor riesgo de enfermedad cardiovascular en mujeres postmenopáusicas que las mujeres premenopáusicas con diabetes mellitus. En un estudio realizado, la terapia hormonal no disminuyó el riesgo de enfermedad cardiovascular en mujeres con diabetes mellitus establecida, lo que sugiere que las hormonas ováricas pueden no estar involucradas (3).

A la diabetes mellitus tipo dos se asocia una dislipidemia típica, caracterizada por hipertrigliceridemia, niveles bajos de colesterol HDL y aumento de la proporción de partículas LDL pequeñas y densas, conocidas por ser más propensas a la oxidación (7).

Otros factores de riesgo de eventos cardiovasculares en pacientes diabéticas incluyen diferencias en las concentraciones de marcadores inflamatorios, poca adherencia al 
tratamiento diabético, y a su vez, estas pacientes tienen mayor probabilidad de presentar múltiples factores de riesgo.

La glucosa postprandial parece ser un predictor más fuerte de enfermedad cardiovascular en mujeres diabéticas que en hombres, mientras que la hemoglobina glucosilada es un mejor predictor de evento cerebrovascular en mujeres diabéticas en comparación con hombres (3).

- Edad de la menarca: un metaanálisis reciente demostró una relación entre la edad de la menarca y el riesgo de padecer todas las causas de muerte, mostrando un $23 \%$ más de riesgo relativo de muerte en mujeres con menarca temprana (<12 años) pero sin protección de menarca tardía.

En un estudio reciente, amplio y prospectivo en el Reino Unido, tanto la menarca temprana (10 años) como la tardía (17 años) se asociaron a un mayor riesgo de enfermedad vascular, especialmente la cardiopatía coronaria isquémica. Los factores genéticos, la obesidad y el tabaquismo pueden, al menos parcialmente, explicar cualquier asociación entre la edad de la menarca y el riesgo cardiovascular (3).

- Otros factores en años de edad reproductiva pueden incrementar el riesgo cardiovascular en la mediana edad incluyen antecedentes de síndrome de ovario poliquístico (SOP), que se asocia comúnmente a resistencia a la insulina, lo que aumenta el riesgo de intolerancia a la glucosa, diabetes mellitus tipo dos y alteraciones en el perfil lipídico que, a su vez, puede conducir a una cardiopatía coronaria ateroesclerótica.
En las mujeres que desarrollan trastornos hipertensivos del embarazo o diabetes gestacional, la disfunción del endotelio vascular ocurre tanto en la circulación uterina como en la materna, lo que puede producir un deterioro de la salud vascular y el inicio de un proceso ateroesclerótico. Los estudios epidemiológicos demuestran una asociación entre la preeclampsia y un mayor riesgo de evento cardiovascular, y a su vez, la diabetes gestacional confiere un riesgo de cuatro a siete veces mayor de desarrollar en el futuro, diabetes mellitus tipo dos y síndrome metabólico (3).

- Edad de la menopausia: la menopausia precoz (antes de los 40 años de edad), ya sea natural o quirúrgica, se ha asociado con un riesgo elevado de enfermedad cardiovascular. Aproximadamente el $1 \%$ de las mujeres de 40 años de edad tienen una insuficiencia ovárica primaria espontánea y presentan una función endotelial deteriorada (3).

La menopausia prematura se ha asociado con un mayor riesgo de enfermedad de las arterias coronarias y, de forma menos constante, con un mayor riesgo de evento cerebrovascular. Además, un análisis de la Iniciativa de Salud de la Mujer encontró una asociación entre la edad menopáusica más temprana y mayor riesgo de insuficiencia cardíaca.

Basados en estos datos, en recientes actualizaciones de las guías del Colegio Americano de Cardiología / Asociación Americana del Corazón, se aprobó el antecedente de menopausia prematura (definida como menopausia antes de los 40 años) para evaluar el riesgo 
cardiovascular y recomendar la prescripción de estatinas a pacientes asintomáticas de mediana edad con riesgo intermedio de enfermedad cardiovascular ateroesclerótica.

En un estudio de cohorte en el cual participaron 144260 pacientes de 40 a 69 años, se demostró que las mujeres postmenopáusicas, naturales y quirúrgicas antes de los 40 años, presentan un aumento moderado, pero estadísticamente significativo de riesgo de una combinación de enfermedades cardiovasculares. En análisis secundarios, la menopausia prematura fue asociada con enfermedad arterial coronaria, insuficiencia cardíaca, estenosis aórtica, fibrilación auricular y tromboembolismo venoso, así como hipertensión arterial, hiperlipidemia y diabetes tipo dos, con mayor riesgo en edades de presentación más tempranas (9). El Consejo Nacional de Investigación Médica y de Salud de Australia (NHMRC, por sus siglas en inglés) realizó diferentes estudios observacionales en 301438 mujeres, de las cuales, un 4.3\% desarrolló enfermedad cardiovascular después de la menopausia, y de este porcentaje, un $3.1 \%$ desarrolló cardiopatía isquémica coronaria y un $1.4 \%$ presentó evento cerebrovascular. El riesgo de enfermedad cardiovascular fue mayor en mujeres con menopausia precoz (<40 años), menopausia temprana (40-44 años) y relativa menopausia temprana (45-49 años) en comparación con las mujeres con menopausia a la edad de 50-51 años. Por consiguiente, se concluyó que en comparación a mujeres con menopausia a la edad de 50-51 años, las mujeres con menopausia precoz o temprana presentan un mayor riesgo de enfermedad cardiovascular antes de los 60 años, pero no después de los 70 años (1).

- Síntomas de la menopausia: los trastornos de la menopausia se pueden dividir en dos grupos principales:

- Síntomas a corto plazo: los más comunes son los sofocos y sudoración nocturna, también conocidos como síntomas vasomotores.

- Síntomas a largo plazo: incluyen enfermedades cardiovasculares, osteoporosis y trastornos de deterioro cognitivo y del estado de ánimo.

Estos trastornos son experimentados por aproximadamente el $40 \%$ de los casos de perimenopausia y menopausia en mujeres en todo el mundo $(2,3,10)$.

Durante muchos años, se pensó que los síntomas a corto plazo y las enfermedades crónicas se producían de forma independiente sin un vínculo causal entre ellos. Sin embargo, existe evidencia que demuestra que lo síntomas de la menopausia, en particular los síntomas vasomotores, pueden considerarse precursores 0 biomarcadores de enfermedades crónicas (10).

Los factores de riesgo tradicionales como una disminución de la tolerancia a la glucosa, aumento de la presión arterial y disfunción endotelial, toma importancia en la transición a la menopausia y posterior a ella, lo cual, en parte explica el aumento de riesgo cardiovascular (2). Los síntomas vasomotores ocurren con mayor frecuencia en mujeres con menopausia prematura (antes de los 40 años de edad), y las mujeres con 
menopausia prematura inducida médicamente (ooforectomía, quimioterapia o radioterapia) pueden experimentar síntomas más graves.

Los síntomas vasomotores son eventos endocrinos y / o termorreguladores que se originan en el hipotálamo como resultado de la disminución de las hormonas ováricas. Las mujeres con sofocos intensos pueden tener un aumento del tono simpático con afectación vascular. Sin embargo, los sofocos representan un fenómeno multicausal complejo y reflejan una combinación de sistemas interconectados que incluyen bases genéticas, dieta, uso de medicamentos, influencias culturales, entre otros (10).

En un metaanálisis reciente, se demostró que los síntomas vasomotores y no vasomotores están relacionados con un incremento del riesgo cardiovascular. Existen hallazgos que indican que las mujeres postmenopáusicas con síntomas vasomotores tienen una mayor prevalencia de factores de riesgo cardiovascular como aumento de presión arterial y alteración del perfil lipídico. Por otra parte, la depresión y la ansiedad pueden resultar de una activación autonómica, que produce un aumento de la presión arterial y la frecuencia cardíaca, además de una disfunción endotelial. Asimismo, puede resultar afectada la coagulación al aumentar la actividad plaquetaria y la viscosidad plasmática (2).

En un estudio observacional en el cual participaron $590 \quad$ mujeres perimenopáusicas, se concluyó que la asociación de síntomas climatéricos con algunos factores de riesgo cardiovascular, puede revelar una vulnerabilidad general a las fluctuaciones de esteroides gonadales de la perimenopausia.

Por otro lado, también puede indicar una relación causa-efecto. Un incremento de la actividad catecolaminérgica y la modificación del péptido relacionado con la calcitonina representan mecanismos que vinculan los síntomas vasomotores con un mayor riesgo cardiovascular. Asimismo, existe un aumento de cortisol y, como consecuencia, resistencia a la insulina y alteraciones del metabolismo de los lípidos. Estas modificaciones bioquímicas comienzan a ocurrir muy temprano, durante la perimenopausia (11).

En diferentes estudios observacionales donde se incluyeron $19 \quad 667$ participantes, se demostró que las mujeres con sofocos, en comparación con las que no los presentaban, tendían a presentar niveles significativamente más altos de presión arterial sistólica y mayores probabilidades de padecer hipertensión arterial. Del mismo modo, las mujeres que reportaron sudores nocturnos en comparación con aquellas que no lo hicieron, tuvieron niveles significativamente más altos de presión arterial sistólica y diastólica, colesterol total y mayor índice de masas corporal (12).

- Menopausia quirúrgica: la histerectomía es uno de los procedimientos quirúrgicos más comunes en mujeres, sin embargo, existe una asociación entre el procedimiento y las enfermedades cardiovasculares, y esto se debe en parte, a un aumento de la inflamación, que, a su vez, resulta en un aumento de la rigidez de la vasculatura $y$, por consiguiente, un mayor riesgo de enfermedades cardiovasculares. 
El riesgo cardiovascular asociado a la histerectomía (con ovarios intactos) se ha atribuido a la menopausia temprana relacionada a una insuficiencia ovárica prematura por alteración del flujo sanguíneo ovárico. Sin embargo, un estudio de La Iniciativa de Salud de las Mujeres (WHI, por sus siglas en inglés) reveló que la histerectomía (incluyendo ooforectomía bilateral o unilateral) sin antecedentes de uso de terapia de reemplazo hormonal (TRH) se asoció a enfermedad arterial coronaria subclínica independiente de los factores de riesgo tradicionales de enfermedad cardiovascular.

En un estudio transversal que se realizó con el objetivo de determinar la asociación entre la rigidez arterial y la menopausia, se demostró que la velocidad de onda de pulso carotídeo femoral y la velocidad de onda de pulso brazo - tobillo, fueron significativamente mayores en mujeres con menopausia quirúrgica en comparación con las mujeres del grupo de menopausia natural y del premenopáusico. La rigidez de las arterias de mediano y gran calibre es un mecanismo importante por el cual la histerectomía aumenta el riesgo de enfermedad cardiovascular en mujeres postmenopáusicas (13).

\section{TERAPIA DE REEMPLAZO HORMONAL}

El cese del desarrollo de los folículos ováricos relacionado con la edad y la extirpación quirúrgica de los ovarios va seguida de una disminución de los estrógenos circulantes, especialmente el estradiol.

Esta disminución de estrógenos produce una serie de síntomas vasomotores, trastornos del sueño, depresión, desequilibrio metabólico, disminución de la masa mineral ósea, disminución de la turgencia de la piel, entre otros. Por esta razón, muchas mujeres se benefician del uso del tratamiento de reemplazo hormonal $(\mathrm{TRH})$, solo con estrógenos para las pacientes a las que se les ha extirpado los ovarios y el útero, o estrógeno más una progestina para aquellas que aún conservan el endometrio (14).

Alrededor de 40 estudios observacionales de cohorte o de casos y controles han demostrado en forma consistente que la terapia de reemplazo hormonal reduce en $30-50 \%$ la incidencia de enfermedad arterial coronaria y la mortalidad cardiovascular en la postmenopausia, especialmente si se administra a mujeres más jóvenes.

El ensayo clínico aleatorizado de La Iniciativa de Salud de las Mujeres (WHI, por sus siglas en inglés) y el estudio ELITE (Early Versus Late Intervention Trial with Estradiol) demostraron que, comenzar el tratamiento de reemplazo hormonal dentro de los 5 a 10 años posteriores a la menopausia es fundamental para el éxito de la cardioprotección en mujeres postmenopáusicas. Debe destacarse que el riesgo cardiovascular en el estudio WHI (enfermedad coronaria, evento cerebrovascular y tromboembolismo venoso) solo se incrementó en el tratamiento combinado con estrógenos y progestinas orales, pero, a excepción del riesgo cerebrovascular, no se presentó en el grupo que utilizó estrógenos solos en mujeres histerectomizadas, lo que pone en evidencia el potencial efecto negativo de la progestina utilizada, la medroxiprogesterona.

Un análisis posterior demostró que, en el caso de la terapia combinada, el aumento de riesgo de enfermedad arterial coronaria se hacía significativo solo en el grupo etario de 
70-79 años y no en las mujeres de menor edad (4).

Por otra parte, una revisión sistemática reciente, concluyó que las pacientes que iniciaron el tratamiento en menos de 10 años de la menopausia, presentaron una menor mortalidad y una menor incidencia de enfermedad coronaria, sin embargo, aún tenían un mayor riesgo de tromboembolismo venoso en comparación con placebo o ningún tratamiento. En el grupo de las que iniciaron tratamiento después de 10 años de la menopausia, hubo evidencia de que el tratamiento tuvo poco efecto sobre la mortalidad e incidencia de enfermedad coronaria, no obstante, hubo un mayor riesgo de evento cerebrovascular y tromboembolismo venoso (15).

Este fenómeno dependiente de la edad se ha denominado "hipótesis del tiempo de uso", que postula que, en mujeres más jóvenes, generalmente menores de 60 años de edad, con un sistema arterial más sano, la terapia de reemplazo hormonal favorecería la cardioprotección por los múltiples mecanismos enunciados, y el inicio de terapia de reemplazo hormonal en mujeres mayores de 60 años o con más de 10 años de postmenopausia; quienes podrían ya presentar placas ateroescleróticas, puede asociarse a un aumento de la expresión de las metaloproteinasas inducido por los estrógenos causando una disrupción de la capa fibrosa y una ruptura de la placa; además, el efecto protrombótico, exclusivo de la vía oral de la administración de los estrógenos, podría favorecer fenómenos de trombosis arterial y venosa en este grupo etario (4).

De esta forma, según los datos de la WHI, se ha evidenciado que el inicio del tratamiento hormonal en la primera década después de la menopausia es seguro y eficaz, incluso en mujeres que se sometieron a una menopausia quirúrgica (14), sin embargo, en mujeres que inician la TRH después de los 60 años, podría asociarse a un aumento del riesgo cardiovascular (4).

\section{DISCUSIÓN}

Se puede determinar, tras la revisión bibliográfica realizada, un evidente incremento del riesgo cardiovascular en la mujer postmenopáusica, siendo este aumento mayor en los casos de menopausia precoz (1), además, se logra establecer el importante rol que juegan las hormonas; tanto los estrógenos como la testosterona, en cuanto a protección y aumento de riesgo cardiovascular respectivamente (6), no obstante, se ha evidenciado también que el aumento del riesgo cardiovascular en la mujer postmenopáusica no depende únicamente de las hormonas endógenas, sino que se presentan una multitud de factores, en su mayoría prevenibles, tales como el tabaquismo, el aumento del colesterol LDL y de triglicéridos, un inadecuado manejo de enfermedades crónicas como la diabetes mellitus y la hipertensión arterial; asimismo se exponen otros factores de riesgo, como es el caso de la menopausia prematura por insuficiencia ovárica espontánea o quirúrgica (9), que no son prevenibles, sin embargo, también influyen de forma importante, acrecentando el riesgo cardiovascular en mujeres postmenopáusicas.

No obstante, no solo se evalúa el aumento del riesgo cardiovascular asociado a la menopausia en todas sus formas y presentaciones, sino que también se logran establecer diferentes medidas que se ha demostrado, en múltiples ensayos, que impactan positivamente en la calidad y en la esperanza de vida, respecto al campo 
cardiovascular, principalmente el uso de terapia hormonal antes de los 10 años posteriores a la presentación de la menopausia (9).

\section{CONCLUSIÓN}

Se presentan múltiples factores que aumentan el riesgo real de enfermedad cardiovascular en mujeres postmenopáusicas, entre ellos se concluyen los siguientes: el tabaquismo, la hipertensión arterial, las dislipidemias, la diabetes mellitus tipo dos, la edad de la menarca y de la menopausia, y los síntomas vasomotores (3), y factores de riesgo tradicionales del periodo de transición, tales como la disminución de la tolerancia a la glucosa, el aumento de la presión arterial y la disfunción endotelial. Asimismo, se exponen otros factores, como la disfunción ovárica primaria y la menopausia inducida quirúrgicamente, que suelen adelantar todos los procesos fisiológicos de una menopausia natural, resultando en un importante aumento del riesgo cardiovascular.
Se logra establecer por tanto, una clara asociación de aumento de casos de enfermedades cardiovasculares en mujeres después de la menopausia, con respecto a aquellas que no han presentado la misma, este riesgo se puede atribuir a múltiples factores endógenos, patológicos, ambientales y genéticos, que pueden llegar a ser en su mayoría prevenibles, con un estilo de vida saludable, evitando el tabaquismo, realizando una adecuada actividad física, monitorizando los niveles de marcadores químicos séricos como la glucosa, colesterol LDL y HDL, triglicéridos, así como un manejo de cifras de presión arterial adecuadas y control de enfermedades crónicas. También en esta misma línea, el presente trabajo, logra concluir que la terapia de reemplazo hormonal se presenta, siempre y cuando se utilice bajo una estricta supervisión médica y antes de los diez primeros años posteriores a la menopausia (15), como un método seguro y eficaz para disminuir el riesgo cardiovascular en mujeres post menopáusicas.

\section{REFERENCIAS}

1. Zhu D, Chung HF, Dobson AJ, Pandeya N, Giles GG, Bruinsma F, et al. Age at natural menopause and risk of incident cardiovascular disease: a pooled analysis of individual patient data. Lancet Public Health 2019; 4: e55364. https://doi.org/10.1016/S2468-2667(19)30155-0

2. Muka T, Oliver-Williams C, Colpani V, Kunutsor S, Chowdhury S, et al. (2016) Association of Vasomotor and Other Menopausal Symptoms with Risk of Cardiovascular Disease: A Systematic Review and Meta-Analysis. PLOS ONE 11(6): e0157417. https://doi.org/10.1371/journal.pone.0157417

3. P. Collins, C. M. Webb, T. J. de Villiers, J. C. Stevenson, N. Panay \& R. J. Baber (2016) Cardiovascular risk assessment in women - an update, Climacteric, 19:4, 329-336. https://doi.org/10.1080/13697137.2016.1198574

4. Arteaga Urzúa E. Menopausia y riesgo cardiovascular. Rev Med Chil. 2016 Nov;144(11):1375-1376. http://dx.doi.org/10.4067/S0034-98872016001100001

5. Bernhardt L, Lawson CA. Early menopause and risk of cardiovascular disease: an issue for young women. Lancet Public Health. 2019 Nov;4(11): e539-e540. https://doi.org/10.1016/ S2468-2667(19)30184-7 
6. Zhao D, Guallar E, Ouyang P, Subramanya V, Vaidya D, Ndumele CE, Lima JA, Allison MA, Shah SJ, Bertoni AG, Budoff MJ, Post WS, Michos ED. Endogenous Sex Hormones and Incident Cardiovascular Disease in PostMenopausal Women. J Am Coll Cardiol. 2018 Jun 5;71(22):2555-2566. https://doi.org/10.1016/.j.jacc.2018.01.083

7. Fonseca, M.I.H., da Silva, I.T. \& Ferreira, S.R.G. Impact of menopause and diabetes on atherogenic lipid profile: is it worth to analyse lipoprotein subfractions to assess cardiovascular risk in women?. Diabetol Metab Syndr 9, 22 (2017). https://doi.org/10.1186/s13098-017-0221-5

8. de Kat, A.C., Dam, V., Onland-Moret, N.C. et al. Unraveling the associations of age and menopause with cardiovascular risk factors in a large population-based study. BMC Med 15, 2 (2017). https://doi.org/10.1186/s12916-016-0762-8

9. Honigberg MC, Zekavat SM, Aragam K, et al. Association of Premature Natural and Surgical Menopause With Incident Cardiovascular Disease. JAMA. 2019;322(24):2411-2421. https://doi.org/10.1001/jama.2019.19191

10. Biglia N, Cagnacci A, Gambacciani M, Lello S, Maffei S, Nappi RE. Vasomotor symptoms in menopause: a biomarker of cardiovascular disease risk and other chronic diseases? Climacteric. 2017 Aug;20(4):306-312. http://dx.doi.org/10.1080/13697137.2017.1315089

11. Cagnacci A, Palma F, Romani C, Xholli A, Bellafronte M, Di Carlo C. Are climacteric complaints associated with risk factors of cardiovascular disease in peri-menopausal women?. Gynecological Endocrinology 2015; 31:5, 359362. https://doi.org/10.3109/09513590.2014.998188

12. Franco OH, Muka T, Colpani V, Kunutsor S, Chowdhury S, Chowdhury R, Kavousi M. Vasomotor symptoms in women and cardiovascular risk markers: Systematic review and meta-analysis. Maturitas 81 (2015) 353-361. http://dx.doi.org/10.1016/j.maturitas.2015.04.016

13. Abbas SZ, Sangawan V, Das A, Pandey AK. Assessment of Cardiovascular Risk in Natural and Surgical Menopause. Indian J Endocrinol Metab. 2018 Mar-Apr;22(2):223-228. https://doi.org/10.4103/2230-8210.232384

14. Naftolin F, Friedenthal $J$, Nachtigall $R$ and Nachtigall L. Cardiovascular health and the menopausal woman: the role of estrogen and when to begin and end hormone treatment. F1000Research 2019, 8(F1000 Faculty Rev):1576. https://doi.org/10.12688/f1000research.15548.1

15. Boardman HMP, Hartley L, Eisinga A, Main C, Roqué i Figuls M, Bonfill Cosp X, Gabriel Sanchez R, Knight B. Hormone therapy for preventing cardiovascular disease in post-menopausal women. Cochrane Database of Systematic Reviews 2015, Issue 3. Art. No.: CD002229. https://doi.org/10.1002/14651858.CD002229.pub4 